\title{
Double parton correlations in Light-Front constituent quark models
}

\author{
Matteo Rinaldi ${ }^{1, a}$, Sergio Scopetta ${ }^{2, b}$, Marco Traini $^{3, c}$, and Vicente Vento ${ }^{4, d}$ \\ ${ }^{1}$ Dipartimento di Fisica e Geologia, Università degli Studi di Perugia, and INFN, sezione di Perugia, via A. Pascoli 06100 Perugia, \\ Italy \\ ${ }^{2}$ Dipartimento di Fisica e Geologia, Università degli Studi di Perugia, and INFN, sezione di Perugia, via A. Pascoli 06100 Perugia, \\ Italy \\ ${ }^{3}$ Dipartimento di Fisica, Università degli studi di Trento, and INFN-TIFPA, via Sommarive 14, I - 38123 Povo (Trento), Italy \\ ${ }^{4}$ Departament de Fisica Teòrica, Universitat de València and Institut de Fisica Corpuscular, Consejo Superior de Investigaciones \\ Científicas, 46100 Burjassot (València), Spain
}

\begin{abstract}
Double parton distribution functions (dPDF) represent a tool to explore the 3D proton structure. They can be measured in high energy proton-proton and proton nucleus collisions and encode information on how partons inside a proton are correlated among each other. dPFDs are studied here in the valence quark region, by means of a constituent quark model, where two particle correlations are present without any additional prescription. This framework allows to understand the dynamical origin of the correlations and to clarify which, among the features of the results, are model independent. Use will be made of a relativistic light-front scheme, able to overcome some drawbacks of the previous calculation. Transverse momentum correlations, due to the exact treatment of the boosts, are predicted and analyzed. The role of spin correlations is also shown. Due to the covariance of the approach, some symmetries of the dPDFs are seen unambigously. For the valence sector, also the study of the QCD evolution of the model results, which can be performed safely thanks to the property of good support, has been also completed.
\end{abstract}

\section{Introduction}

In the last few years, the study of the contribution of multiple parton interactions (MPI) to high energy hadronhadron cross sections has become an important issue. In these processes, more than one parton of a hadron can interact with partons of the other colliding hadron. Even if the MPI contribution is suppressed by a power of $\Lambda_{Q C D}^{2} / Q^{2}$ with respect to the single parton interaction, where $Q$ is the center-mass energy, it has been already observed (see, e.g., Ref. [1]). These events can be very important as a background for the search of new Physics, e.g., at the LHC. In our analysis we focus actually on the possibilities offered by double parton scattering (DPS), which can be observed in several channels, e.g., $W W$ with dilepton productions and double Drell-Yan processes (see, Refs. [2-6] for recent reviews), to unveil the 3D nucleon structure. At the LHC, DPS, already observed some years ago [7], represents also a background for the Higgs production in several channels.

The DPS cross section is written, following the seminal idea of Ref. [8], in terms of the dPDFs, $F_{i j}\left(x_{1}, x_{2}, \vec{z}_{\perp}, \mu\right)$, which describe the joint probability of finding two partons of flavors $i, j=q, \bar{q}, g$ with longitu-

\footnotetext{
a e-mail: matteo.rinaldi@pg.infn.it

be-mail: sergio.scopetta@pg.infn.it

ce-mail: traini@science.unitn.it

de-mail: vicente.vento@uv.es
}

dinal momentum fractions $x_{1}, x_{2}$ and separation $\vec{z}_{\perp}$ in the transverse plane inside the hadron:

$$
\begin{aligned}
d \sigma= & \frac{1}{S} \sum_{i, j, k, l} \int d \vec{z}_{\perp} F_{i j}\left(x_{1}, x_{2}, \vec{z}_{\perp}, \mu\right) F_{k l}\left(x_{3}, x_{4}, \vec{z}_{\perp}, \mu\right) \\
& \times \quad \hat{\sigma}_{i k}\left(x_{1} x_{3} \sqrt{s}, \mu\right) \hat{\sigma}_{j l}\left(x_{2} x_{4} \sqrt{s}, \mu\right) .
\end{aligned}
$$

The cross sections $\hat{\sigma}$ refer to the hard parton scattering, occurring at short-distance, $S$ is a symmetry factor, present if identical particles appear in the final state and $\mu$ is the renormalization scale. The latter is taken here, for simplicity, to be the same for both partons.

It is worth to notice that usually, for the evaluation and simulation of the DPS contributions to proton-proton scattering at the LHC kinematics, the following approximations are often adopted for the dPDFs:

$$
\begin{aligned}
F_{i j}\left(x_{1}, x_{2}, \vec{z}_{\perp}, \mu\right) & =q_{i}\left(x_{1}, \mu\right) q_{j}\left(x_{2}, \mu\right) T\left(\vec{z}_{\perp}, \mu\right) \\
& \times \theta\left(1-x_{1}-x_{2}\right)\left(1-x_{1}-x_{2}\right)^{n}
\end{aligned}
$$

i.e., dPDFs are assumed to be completely factorized. In particular the $\vec{z}_{\perp}$ and $x_{1}-x_{2}$ dependences are factorized and that the latter dependence can be expressed through the standard one-body parton distribution functions (PDF), $q(x)$. In other words, possible double parton correlations between the two interacting partons are neglected. Moreover, dPDFs are non perturbative quantities in QCD and 
they cannot be easily evaluated from the theory. In the case of PDFs, a useful procedure for the estimate of dPDFs is their calculation at the hadronic scale, $Q_{0} \sim \Lambda_{Q C D}$, by means of quark models. In order to compare the obtained outcomes with future data taken at high energy scales, $Q>Q_{0}$, it is then necessary to perform the perturbative QCD (pQCD) evolution of the model calculations, using the dPDF evolution equations, which are known since a long time ago $[9,10]$. The idea supporting our analysis is that, thanks to this procedure, future data of the DPS processes could be guided, in principle, by model calculations. In particular, our interest is focused on the understanding of the role of double parton correlations (DPCs) in the proton, in order to verify, as a first step, the validity of the approximations Eq. (2), often used for simulations of data analyses. Moreover, DPCs effects, cannot be neglected in principle (see Refs. [11, 12] for recent contribution on this subject); however, DPCs are non perturbative effects in QCD and they can not be easily evaluated from the theory. In order to understand the role of DPCs in the proton structure and in the dPDFs, the use of quark models, where DPCs are naturally included into the scheme, can be very useful. Thanks to this procedure, in the kinematical regions where these predictions are reliable, the assumptions Eq. (2) can be properly tested.

The first model evaluations of dPDFs have been the ones in Refs. [13, 14]. In the first scenario use has been made of a the MIT bag model in the cavity-approximation, properly modified in order to introduce double parton correlations. In the second case dPDFs have been calculated in a non relativistic (NR) constituent quark model (CQM) framework, since CQMs, in the valence region, reproduce the gross features of PDF data and give reasonable predictions of generalized parton distribution functions (GPDs) and transverse momentum dependent parton distributions (TMDs) (see, e.g., Refs. [15-17]). These expectations motivated the analysis of Refs. [14, 18]. The main results found in Refs. $[13,14]$ are that, in the valence quark region, the approximations described in Eq. (2) are badly violated. In this CQM picture, where dynamical double correlations are present into the scheme, the origin of this violation can be properly understood.

One should notice that both the analyses of Refs. $[13,14]$ have inconsistencies. First, they predict a wrong support, i.e., dPDFs do not vanish in the non physical region, $x_{1}+x_{2}>1$. Moreover, as already pointed out, in order to obtain some information on dPDFs where LHC data will be taken, i.e., at small values of $x$ and at high $Q^{2}$, the pQCD evolution of the calculated dPDFs is necessary. In a recent paper [18], we calculated dPDFs including relativity through a fully Poincaré covariant Light-Front (LF) approach. Thanks to this treatment it is possible in general to study strongly interacting systems with a fixed number of on-shell constituents (see Refs. [19, 20] for comprehensive reviews). In this framework, which has been extensively used for calculations of hadron structure observables (see, e.g., Refs. [21-23]), some symmetries of dPDFs are correctly restored and the bad support problem is fixed. This helps in turn to have a precise pQCD evo- lution of the dPDFs. The results of this analysis will be summarized in the following sections.

\section{Light-Front CQM and dPDFs}

In this section, details of the calculations of dPDFs, within the LF approach will be introduced. In particular, the factorized ansatz Eq. (2), adopted for the quantities under scrutiny here, will be studied in a relativistic scenario. In order to have a reliable description of dPDFs, defined in the non perturbative region of QCD, use has been made of the Light-Front approach, which is well suited, as already said, for studies of hadronic processes at high momentum transfer. Among all the useful properties of this approach, let us summarize the ones which are appealing for our aim. First of all, one can obtain a fully Poincaré covariant description of relativistic strongly interacting systems with a fixed number of on-shell constituents. Moreover, LF boosts and the "plus" components of momenta $\left(a^{+}=a_{0}+a_{3}\right)$ are kinematical operators and, being the LF hypersurface, the one where the initial conditions of the system are fixed, tangent to the light-cone, the kinematics of DIS processes is obtained naturally.

In the present analysis, the main quantity of interest is the Fourier- transform of the dPDF defined in Eq. (1):

$F_{i j}^{\lambda_{1}, \lambda_{2}}\left(x_{1}, x_{2}, \vec{k}_{\perp}\right)=\int d \vec{z}_{\perp} e^{i \vec{z}_{\perp} \cdot \vec{k}_{\perp}} F_{i j}^{\lambda_{1}, \lambda_{2}}\left(x_{1}, x_{2}, \vec{z}_{\perp}\right)$.

The dPDF here introduced depends on the parton flavors $i$ and $j$ and on their helicities $\lambda_{i(j)}$, respectively. Since dPDFs will be calculated here by means of a CQM, it is necessary to find useful expressions for the dPDFs, by means of the LF approach. One needs therefore to start from the light-cone correlator which formally defines the dPDF in quantum field theory [4]:

$$
\begin{aligned}
F_{i j}^{\lambda_{1}, \lambda_{2}}\left(x_{1}, x_{2}, \vec{z}_{\perp}\right) & =\left(-4 \pi P^{+}\right) \sum_{\lambda} \int d \vec{z}_{\perp} e^{i z_{\perp} \cdot \vec{k}_{\perp}} \int\left[\prod_{l}^{3} \frac{d z_{l}^{-}}{4 \pi}\right] \\
& \times e^{i x_{1} P^{+} z_{1}^{-} / 2} e^{i x_{2} P^{+} z_{2}^{-} / 2} e^{-i x_{1} P^{+} z_{3}^{-} / 2} \\
& \times\langle\lambda, \vec{P}=\overrightarrow{0}| \hat{O}_{i}^{1}\left(z_{1}^{-} \frac{\bar{n}}{2}, z_{3}^{-} \frac{\bar{n}}{2}+\vec{z}_{\perp}\right) \\
& \times \hat{O}_{j}^{2}\left(z_{2}^{-} \frac{\bar{n}}{2}+\vec{z}_{\perp}, 0\right)|\vec{P}=\overrightarrow{0}, \lambda\rangle
\end{aligned}
$$

where, for generic 4-vectors $z$ and $z^{\prime}$, the operator

$$
\hat{O}_{i}^{k}\left(z, z^{\prime}\right)=\bar{q}_{i}(z) \frac{\hbar}{2} \frac{1+\lambda_{k} \gamma_{5}}{2} q_{i}\left(z^{\prime}\right)
$$

has been defined in coordinate space through LF quantized fields of the free quarks. In the above equation, use has been made of the light-like four vector, $\bar{n}=$ $(1,0,0,-1)$, and of the rest frame state of the nucleon with helicity $\lambda,|\vec{P}=\overrightarrow{0}, \lambda\rangle$. By properly extending the procedure described in Refs. [23, 24] for the calculation of GPDs, one can relate dPDFs to the proton wave function, which 
can be calculated in turn through CQM. Details on this calculation scheme can be found in Ref. [18]. In particular, a crucial point of this analysis is the description of the proton state in terms of a sum over partonic Fock states $\left|\tilde{k}_{i}, \lambda_{i}^{f}, \tau_{i}\right\rangle$ of isospin $\tau_{i}$, the so called "LF wave function" (LFWF) representation [20]. Moreover, in the present work, only the first, valence contribution $\mid \overrightarrow{0}, \lambda^{f}$,val $\rangle$ has been taken into account and higher Fock states have been neglected:

$$
\begin{aligned}
\left.|\overrightarrow{0}, \lambda\rangle \simeq \mid \overrightarrow{0}, \lambda^{f}, \text { val }\right\rangle & =\sum_{\lambda_{i}^{f} \tau_{i}} \int\left[\prod_{i=1}^{3} \frac{d x_{i}}{\sqrt{x_{i}}}\right] \delta\left(1-\sum_{i=1}^{3} x_{i}\right)(6) \\
& \times\left[\prod_{i=1}^{3} \frac{d \vec{k}_{i \perp}}{2(2 \pi)^{3}}\right] 2(2 \pi)^{3} \delta\left(\sum_{i=1}^{3} \vec{k}_{i \perp}\right) \\
& \times \psi_{\lambda}^{[f]}\left(\left\{x_{i}, \vec{k}_{i \perp}, \lambda_{i}^{f}, \tau_{i}\right\}\right) \prod_{i=1}^{3}\left|\tilde{k}_{i}, \lambda_{i}^{f}, \tau_{i}\right\rangle,
\end{aligned}
$$

where $\psi^{[f]}$ is the Light-Front proton wave function, in principle solution of a LF Hamiltonian directly related to the QCD lagrangian and the short notation $\left\{\alpha_{i}\right\}$ instead of $\alpha_{1}, \alpha_{2}, \alpha_{3}$ is introduced. This representation of the proton state can be formulated in the canonical Instant-Form as well as in the LF case through the introduction of canonical quark states $\left|\vec{k}_{i}, \lambda_{i}^{c}, \tau_{i}\right\rangle$ :

$$
\begin{aligned}
\left.|\overrightarrow{0}, \lambda\rangle \simeq \mid \overrightarrow{0}, \lambda^{c}, \text { val }\right\rangle & =\sum_{\lambda_{i}^{c} \tau_{i}} \int\left[\prod_{i=1}^{3} d \vec{k}_{i}\right] \delta\left(\sum_{i=1}^{3} \vec{k}_{i}\right) \\
& \times \psi_{\lambda}^{[c]}\left(\left\{\vec{k}_{i}, \lambda_{i}^{c}, \tau_{i}\right\}\right) \prod_{i=1}^{3}\left|\vec{k}_{i}, \lambda_{i}^{c}, \tau_{i}\right\rangle,
\end{aligned}
$$

where here $\psi^{[c]}$ is, instead, the canonical proton wave function. Since $\psi^{[f]}$ is basically unknown, being a solution of the QCD equations of motion, it is worth to construct the Poincaré operators in order to obtain a kinematical link between $\psi^{[f]}$ and $\psi^{[c]}$, being the latter quantity calculable by means of the CQM. Formally, one can always relate free particles states in the LF and in the Instant-Form approaches (see Ref. [19]):

$$
\left|\tilde{k}, \lambda^{f}, \tau\right\rangle=(2 \pi)^{3 / 2} \sqrt{m^{2}+\vec{k}_{i}^{2}} \sum_{\lambda^{c}} D_{\lambda^{f} \lambda^{c}}^{1 / 2}\left(R_{c f}(\vec{k})\right)\left|\vec{k}, \lambda^{c}, \tau\right\rangle,
$$

where the Melosh rotation, which allows to rotate the canonical helicity into the LF spin,

$D_{\mu \lambda}^{1 / 2}\left(R_{c f}\left(\vec{k}_{i}\right)\right)=\left\langle\mu\left|\frac{m+x_{i} M_{0}-i \vec{\sigma}_{i} \cdot\left(\hat{z}_{i} \times \vec{k}_{i \perp}\right)}{\sqrt{\left(m+x_{i} M_{0}\right)^{2}+\vec{k}_{i \perp}^{2}}}\right| \lambda\right\rangle$,

has been introduced through $x_{i}=\frac{k_{i}^{+}}{P^{+}}$, the longitudinal momentum fraction carried by the parton $i$, being $P^{+}$the plus component of the proton momentum, $M_{0}=\sum_{i} \sqrt{m^{2}+\vec{k}_{i}^{2}}$ the total free energy mass of the partonic system and $\mu$ and $\lambda$ generic canonical spins. One can use then the
Bakamjian-Thomas construction of the Poincaré generators in order to extend the relation, Eq. (8), to interacting systems, such as the proton one, so that a kinematical link between the LF and Instant-Form proton state can be found [18, 23]:

$$
\left|\overrightarrow{0}, \lambda^{f}, v a l\right\rangle=\sqrt{M_{0}}(2 \pi)^{3 / 2}\left|\overrightarrow{0}, \lambda^{c}, v a l\right\rangle .
$$

Substituting Eqs. (8) and (10) in Eq. (6), one can find the relation between the LF and the canonical proton wave functions, see Refs. [18, 23]:

$$
\begin{aligned}
\psi_{\lambda}^{[f]}\left(\left\{x_{i}, \vec{k}_{i \perp}, \lambda_{i}^{f}, \tau_{i},\right\}\right) & =2(2 \pi)^{3}\left[\frac{\omega_{1} \omega_{2} \omega_{3}}{M_{0} x_{1} x_{2} x_{3}}\right]^{1 / 2} \\
& \times \prod_{i=1}^{3}\left[\sum_{\lambda_{i}^{c}} D_{\lambda_{i}^{c} \lambda_{i}^{f}}^{* 1 / 2}\left(R_{c f}\left(\vec{k}_{i}\right)\right)\right] \\
& \times \psi_{\lambda}^{[c]}\left(\left\{\vec{k}_{i}, \lambda_{i}^{c}, \tau_{i}\right\}\right) .
\end{aligned}
$$

This is an important result for the present study, because it allows to formally relate the LF proton wave function with a canonical proton one. In principle, the LF proton wave function is a solution of the LF QCD Hamiltonian; here we adopt a phenomenological approach where it is obtained from a CQM, reproducing only part of the symmetries of the theory. Using a lengthy but straightforward procedure, the final expression of the $\mathrm{dPDF}$ is obtained. It reads [18]:

$$
\begin{aligned}
F_{q_{1} q_{2}}^{\lambda_{1}, \lambda_{2}}\left(x_{1}, x_{2}, \vec{k}_{\perp}\right) & =3(\sqrt{3})^{3} \int\left[\prod_{i=1}^{3} d \vec{k}_{i} \sum_{\lambda_{i}^{f} \tau_{i}}\right] \delta\left(\sum_{i=1}^{3} \vec{k}_{i}\right) \\
& \times \Psi^{*}\left(\vec{k}_{1}+\frac{\vec{k}_{\perp}}{2}, \vec{k}_{2}-\frac{\vec{k}_{\perp}}{2}, \vec{k}_{3} ;\left\{\lambda_{i}^{f}, \tau_{i}\right\}\right) \\
& \times \widehat{P}_{q_{1}}(1) \widehat{P}_{q_{2}}(2) \widehat{P}_{\lambda_{1}}(1) \widehat{P}_{\lambda_{2}}(2) \\
& \times \Psi\left(\vec{k}_{1}-\frac{\vec{k}_{\perp}}{2}, \vec{k}_{2}+\frac{\vec{k}_{\perp}}{2}, \vec{k}_{3} ;\left\{\lambda_{i}^{f}, \tau_{i}\right\}\right) \\
& \times \delta\left(x_{1}-\frac{k_{1}^{+}}{P^{+}}\right) \delta\left(x_{2}-\frac{k_{2}^{+}}{P^{+}}\right) .
\end{aligned}
$$

The canonical proton wave function $\psi^{[c]}$ is embedded in the function $\Psi$ here above, which can be written as follows:

$$
\begin{aligned}
\Psi\left(\vec{k}_{1}, \vec{k}_{2}, \vec{k}_{2} ;\left\{\lambda_{i}^{f}, \tau_{i}\right\}\right) & \left.=\prod_{i=1}^{3}\left[\sum_{\lambda_{i}^{c}} D_{\lambda_{i}^{c} \lambda_{i}^{f}}^{* 1 / 2}\left(R_{c f}\left(\vec{k}_{i}\right)\right)\right](13)\right) \\
& \times \psi^{[c]}\left(\left\{\vec{k}_{i}, \lambda_{i}^{c}, \tau_{i}\right\}\right)
\end{aligned}
$$

where $\lambda_{i}^{c}$ and $\tau_{i}$ are the canonical parton helicity and the isospin, respectively. Isospin and spin projection operators are introduced in order to define dynamical correlations for the unpolarized and longitudinal polarized $i$ quark of a given flavor, as follows:

$$
\hat{P}_{u(d)}(i)=\frac{1 \pm \tau_{3}(i)}{2}, \hat{P}_{\lambda_{k}}(i)=\frac{1+\lambda_{k} \sigma_{3}(i)}{2}
$$




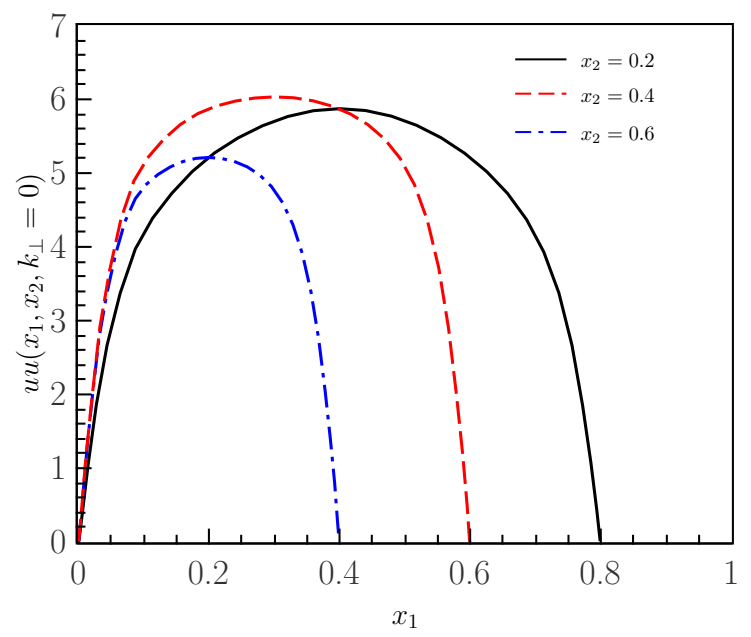

Figure 1. The distribution $u u\left(x_{1}, x_{2}, k_{\perp}\right)$, Eq. (16), for three values of $x_{2}$ and $k_{\perp}=0$.

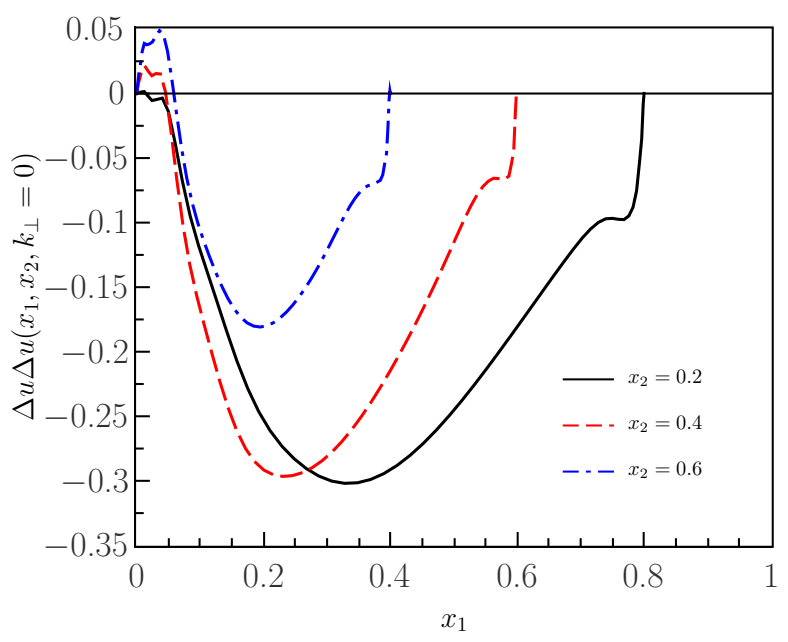

Figure 2. The distribution $\Delta u \Delta u\left(x_{1}, x_{2}, k_{\perp}\right)$, Eq. (17), for three values of $x_{2}$ and $k_{\perp}=0$.

In this work the use of the LF approach is crucial since the plus component of the momenta is kinematical so that, in the intrinsic frame, where $\vec{k}_{1}+\vec{k}_{2}+\vec{k}_{3}=0$, one finds the following condition:

$$
P^{+}=\sum_{i}^{3} k_{i}^{+}=M_{0} .
$$

Thanks to the latter relation, the delta function, defining the longitudinal momentum fractions carried by the partons in Eq. (12), can be easily worked out without any additional approximation, at variance with what happens in the instant form calculations of PDFs and dPDFs (see, e.g., Ref. [14]). As a direct consequence, the bad support problem does not show up in our relativistic calculation. Let us remark again that the main ingredient in Eq. (12) is the canonical proton wave function so that, in order to estimate dPDFs, verifying if the approximations, Eq. (2), hold, use has been made of a relativistic CQM, the so called hyper-central CQM described in Ref. [22]. For the present analysis it is sufficient to know that the proton

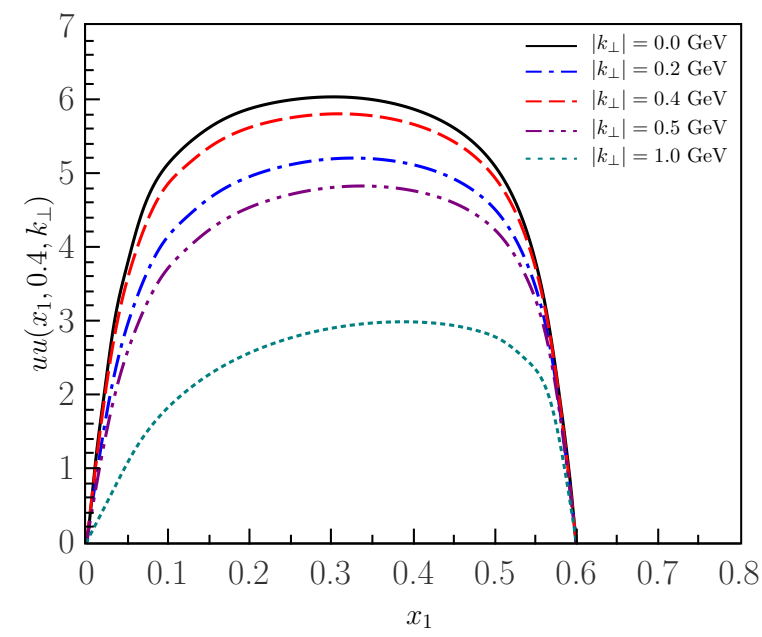

Figure 3. The distribution $u u\left(x_{1}, x_{2}, k_{\perp}\right)$, Eq. (16), for $x_{2}=$ 0.4 and five values of $k_{\perp}$.

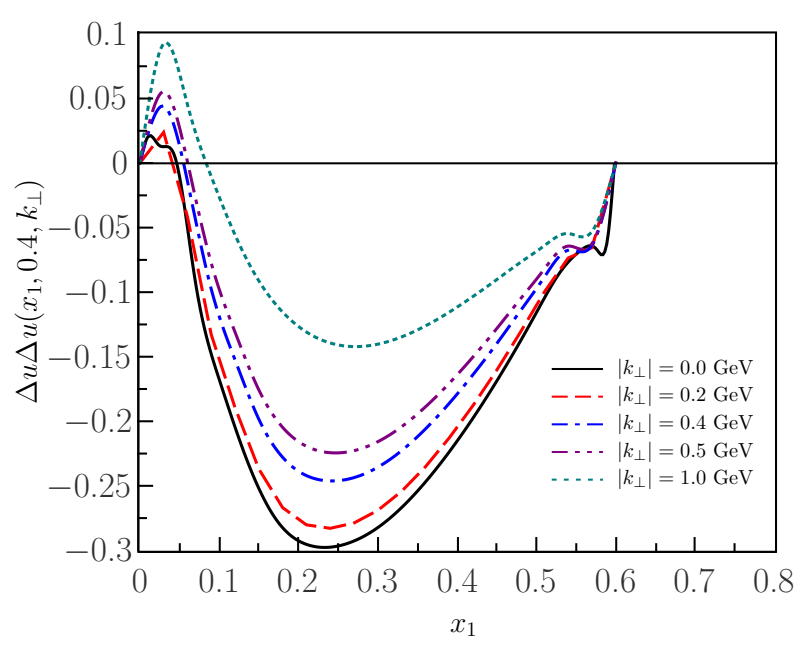

Figure 4. The distribution $\Delta u \Delta u\left(x_{1}, x_{2}, k_{\perp}\right)$, Eq. (17), for $x_{2}=0.4$ and five values of $k_{\perp}$.

wave function can be factorized in terms of the momentum space function and the spin-isospin one. As a first approximation, the latter part will be described within SU(6) symmetry. Moreover, the momentum space function is obtained by solving a relativistic effective Mass equation, described through a potential model in agreement with the Bakamjian-Thomas construction [18, 22]. The choice of this model is motivated by its simplicity and capability to basically reproduce the spectrum of light-hadrons. Indeed it has been already used for the estimate of PDFs and GPDs, see Refs. [22-26]. Moreover, in the dPDFs case, there are not yet data available and model calculations of these quantities could give essential information on their relevant features.

\section{Results of the calculations at the hadronic scale}

In this section the main results, obtained at the hadronic energy scale, $\mu_{0}^{2} \sim 0.1 \mathrm{GeV}^{2}$, will be discussed. In particular, the emphasis of the present analysis is focused on testing the validity of the approximations Eq. (2). To this 


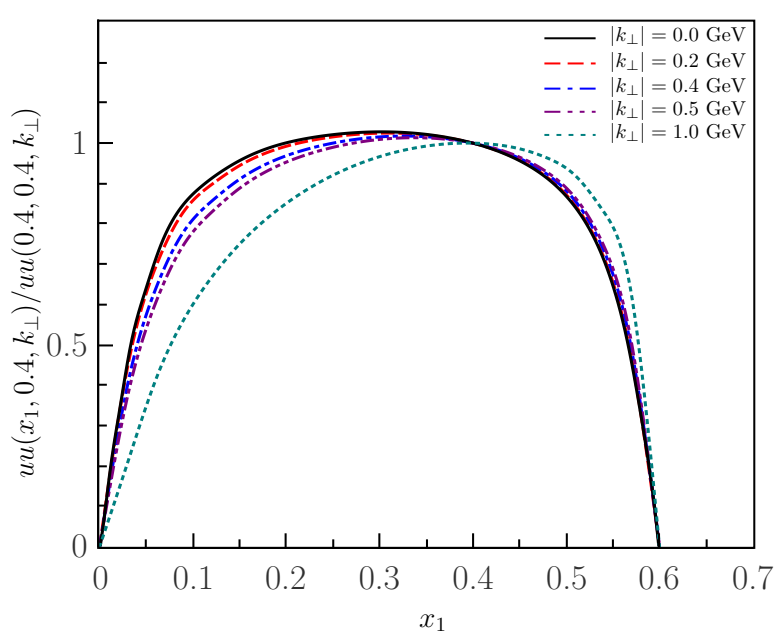

Figure 5. The ratio $r_{1}$, Eq. (18), for five values of $k_{\perp}$.

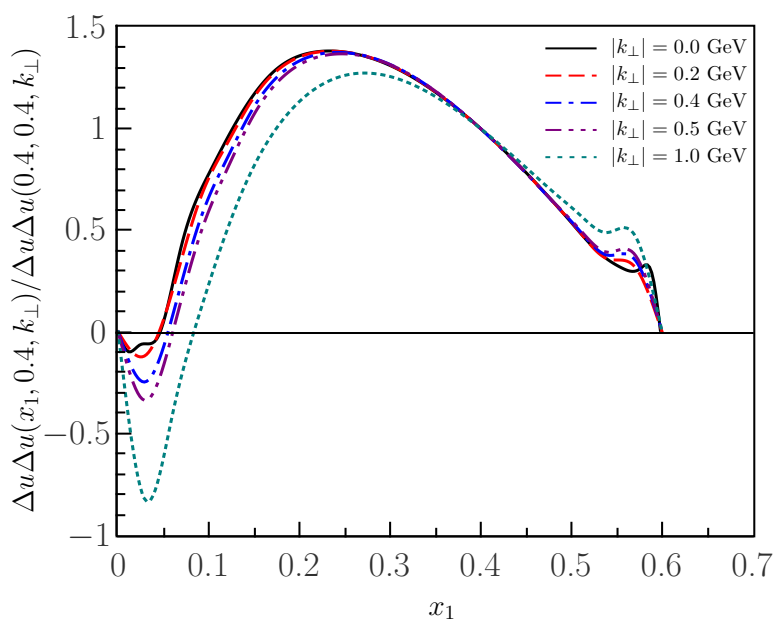

Figure 6. The ratio $r_{2}$, Eq. (19), for five values of $k_{\perp}$.

aim, in this work, two specific combinations of the components of the spin-dependent dPDFs, Eq. (12), for two quarks of flavor $u$, have been evaluated:

$$
\begin{aligned}
u u\left(x_{1}, x_{2}, k_{\perp}\right) & =\sum_{i, j=\uparrow, \downarrow} u_{i} u_{j}\left(x_{1}, x_{2}, k_{\perp}\right), \\
\Delta u \Delta u\left(x_{1}, x_{2}, k_{\perp}\right) & =\sum_{i=\uparrow, \downarrow} u_{i} u_{i}\left(x_{1}, x_{2}, k_{\perp}\right) \\
& -\sum_{i \neq j=\uparrow, \downarrow} u_{i} u_{j}\left(x_{1}, x_{2}, k_{\perp}\right),
\end{aligned}
$$

being these distributions observable, in principle, in processes involving unpolarized protons. In Figs. 1 and 2 the distributions introduced in Eqs. $(16,17)$ are shown for three values of $x_{2}$ and for $k_{\perp}=0$. One can easily realize that the support problem has been solved, i.e., the dPDFs are different from zero only in physical kinematical regions, when $x_{1}+x_{2} \leq 1$. Moreover, thanks to the overcome of the inconsistency present in the canonical calculations of dPDFs in Ref. [13], the symmetry in exchanging $x_{1}$ and $x_{2}$, due to the indistinguishability of the two interacting particles when $k_{\perp}=0$, is restored.

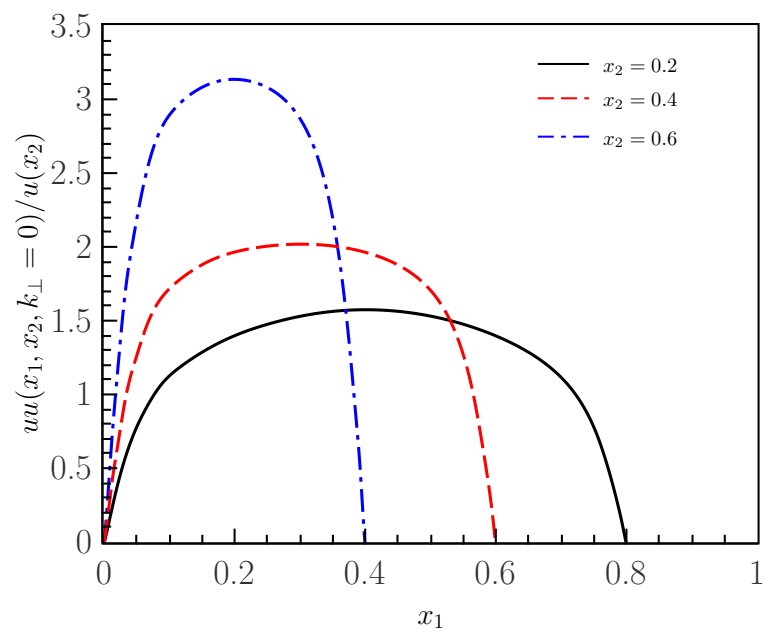

Figure 7. The ratio $r_{3}$, Eq. (20), for three values of $x_{2}$ and $k_{\perp}=0$.

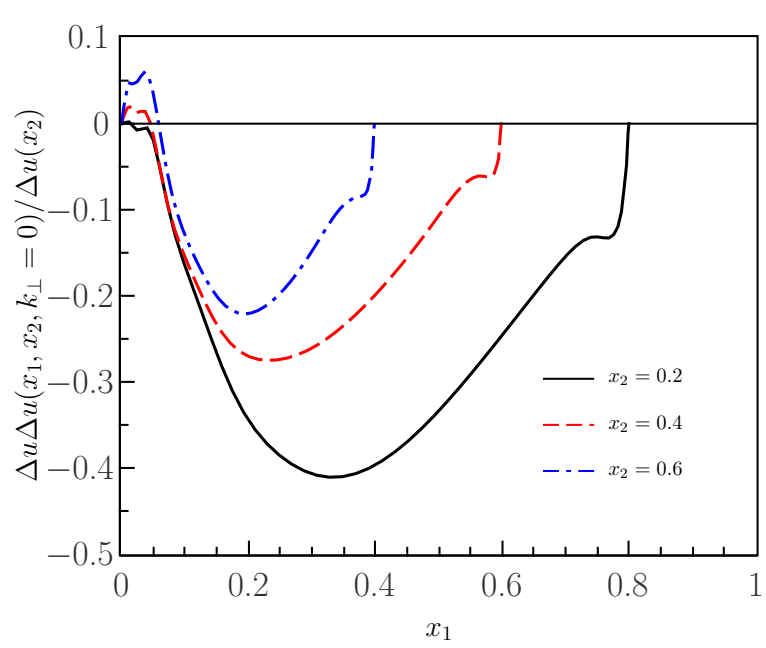

Figure 8. The ratio $r_{4}$, Eq. (21), for three values of $x_{2}$ and $k_{\perp}=0$.

In Figs. 3 and 4 the same distributions are shown for five values of $k_{\perp}$ and for $x_{2}=0.4$. In particular, the decreasing behavior of the dPDFs with respect to the increasing of $k_{\perp}$ is qualitatively similar to the results discussed in Refs. $[13,14]$. In order to study the validity of the approximations Eq. (2), in particular the factorization of dPDFs in the $k_{\perp}$ and $x_{1}, x_{2}$ dependence, the following ratios have been evaluated:

$$
\begin{aligned}
& r_{1}=\frac{u u\left(x_{1}, 0.4, k_{\perp}\right)}{u u\left(0.4,0.4, k_{\perp}\right)}, \\
& r_{2}=\frac{\Delta u \Delta u\left(x_{1}, 0.4, k_{\perp}\right)}{\Delta u \Delta u\left(0.4,0.4, k_{\perp}\right)} .
\end{aligned}
$$

In Figs. 5 and 6 the calculated ratios $r_{1}$ and $r_{2}$ have been shown for five values $k_{\perp}$. As one can see, these quantities vary weakly on $k_{\perp}$ so that a factorized ansatz of dPDFs on $k_{\perp}$ and $x$ dependence is not valid in this approach, as in the cases discussed in Refs. [13, 14]. Let us remark in particular that the amount of this violation is strongly related to the contribution of the Melosh rota- 
tions, present also in the unpolarized case when $k_{\perp} \neq 0$. These kind of relativistic effects are model independent.

In order to study the factorization on the $x_{1}$ and the $x_{2}$ dependences, the following ratios have been introduced:

$$
\begin{aligned}
& r_{3}=\frac{u u\left(x_{1}, x_{2}, k_{\perp}=0\right)}{u\left(x_{2}\right)}, \\
& r_{4}=\frac{\Delta u \Delta u\left(x_{1}, x_{2}, k_{\perp}=0\right)}{\Delta u\left(x_{2}\right)},
\end{aligned}
$$

where the standard PDFs, calculated within the same hyper-central CQM, $u(x)$ and $\Delta u(x)$, in the unpolarized and polarized cases, respectively, have been introduced to analyze if dPDFs can be factorized, in terms of the product of two single PDFs, in this relativistic approach. In Figs. 7 and 8 the ratios $r_{3}$ and $r_{4}$, calculated for three values of $x_{2}$ and for $k_{\perp}=0$, are shown. As one can see, these quantities depend on $x_{2}$, at variance with what would happen if the approximation, Eq. (2), were valid. One can deduce therefore that the dPDFs here calculated are strongly different form the product two single PDFs. These results are in qualitatively agreement with the ones of Refs. [13, 14]. To have more details on the violation on the factorization of longitudinal momentum dependences, the following ratios have been introduced and calculated:

$$
\begin{aligned}
& r_{5}=\frac{2 u u\left(x_{1}, x_{2}, k_{\perp}=0\right)}{u\left(x_{1}\right) u\left(x_{2}\right)}, \\
& r_{6}=\frac{C \Delta u \Delta u\left(x_{1}, x_{2}, k_{\perp}=0\right)}{\Delta u\left(x_{1}\right) \Delta u\left(x_{2}\right)},
\end{aligned}
$$

where:

$$
C=\frac{\left[\int d x \Delta u(x)\right]^{2}}{\int d x_{1} d x_{2} \Delta u \Delta u\left(x_{1}, x_{2}, k_{\perp}=0\right)} .
$$

The single PDFs appear in the denominators of the above equations. Besides, the factors 2 and $C$ are properly inserted in, Eqs. (22) and (23), in order to normalize these ratios in the kinematical regions where the correlations, in the longitudinal momenta, can be neglected, in agreement with the approximation of Eq. (2).

As one can see in Figs. 9 and 10, a factorized form of dPDFs in terms of single PDFs is not supported by the present approach in almost all the kinematical regions where the predictions of CQM calculations are reliable.

\section{4 pQCD evolution of the calculated dPDFs}

A fundamental point discussed in in Ref. [18] is the analysis of the pQCD evolution of our dynamical model of dPDFs. This procedure is essential to relate CQM predictions with future data taken at the LHC or with simulations of DPS process contributions to events in particular channels in $p p$ and $p A$ collisions. For the moment being, the pQCD evolution of dPDFs is performed only in the longitudinal momentum dependence, which means $k_{\perp}=0$, and using the same energy scale

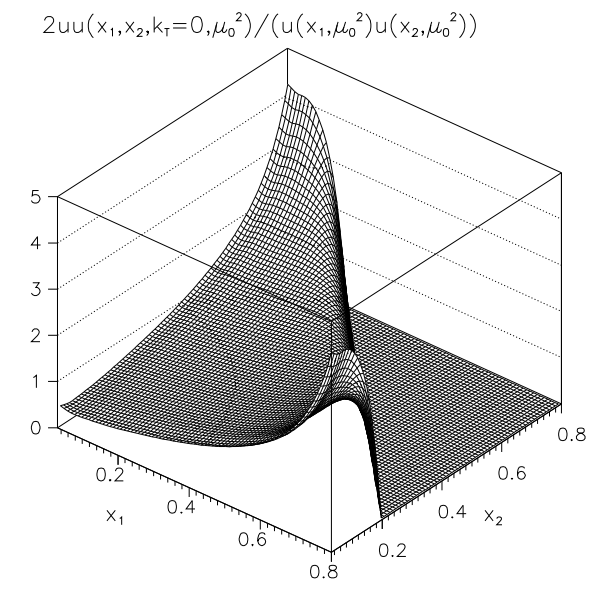

Figure 9. The ratio $r_{5}$, Eq. (22), at the hadronic scale.

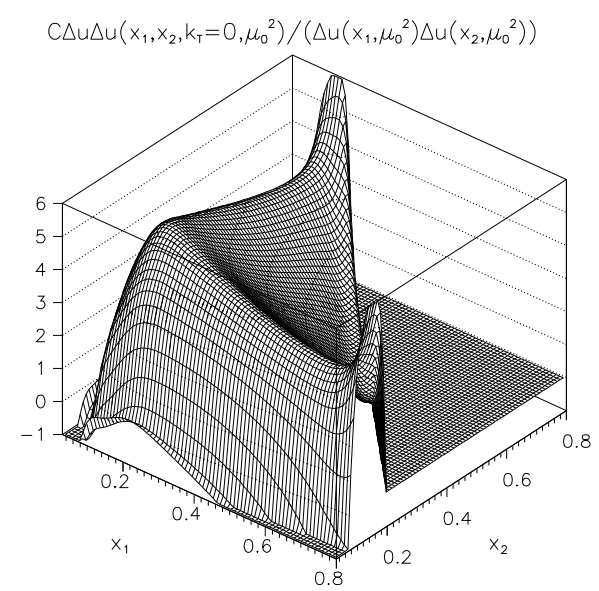

Figure 10. the ratio $r_{6}$, Eq. (23), at the hadronic scale $\mu_{0}$.

for both the acting partons. In these case, the evolution equations are obtained as a proper generalization of the usual Dokshitzer-Gribov-Lipatov-Altarelli-Parisi (DGLAP) ones (see Refs. [9, 10] for details), defined for the evolution of PDFs. In the present analysis only the contribution of the valence, non-singlet sector, has been taken into account in the evolution at Leading-Order, so that one needs to solve only the homogeneous part of of the evolution equations by using the Mellin transformations of dPDFs (see Ref. [18] for details). We notice that, if we use the simple ansatz discussed in Ref. [27] for the evaluation of dPDFs, our code for the $\mathrm{pQCD}$ evolution reproduce the results found and discussed in that paper. After having obtained the $\mathrm{dPDF}$ at a generic high energy scale, e.g., $Q^{2}=10 \mathrm{GeV}^{2}$, using pQCD, the ratios $r_{5}$ and $r_{6}$ have been shown again in Figs. 11 and 12. The most important results of this analysis are that, for small values of $x$, e.g., close to the LHC kinematics, $r_{5} \sim 1$. This means that, in the unpolarized case, dynamical correlations are suppressed after the evolution. Nevertheless, by looking at $r_{6}$, in Fig 6 , it is found that double spin correlations still contribute, even at low $x$. 


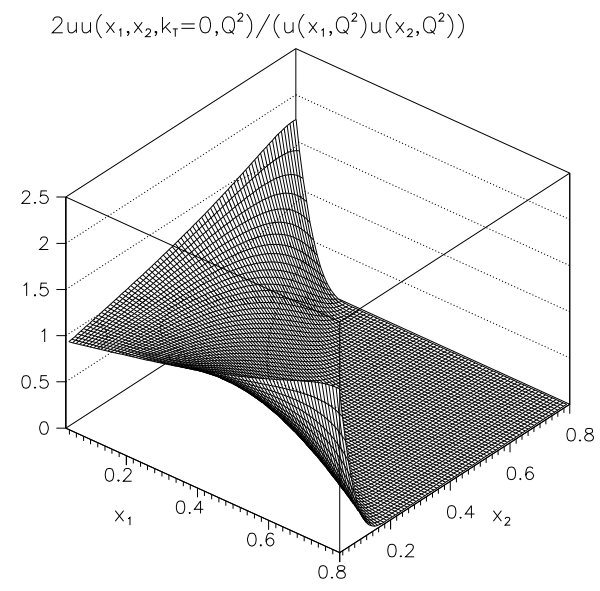

Figure 11. The ratio $r_{5}$, Eq. (22), at a scale $Q^{2}=10 \mathrm{GeV}^{2}$.

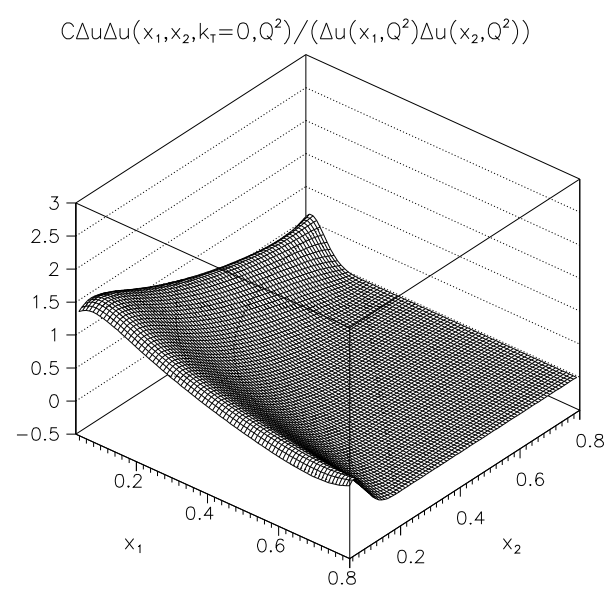

Figure 12. the ratio $r_{6}$, Eq. (23), at a scale $Q^{2}=10 \mathrm{GeV}^{2}$.

\section{Conclusions}

In this work, dPDFs appearing in the DPS cross section have been evaluated within a LF CQM. Fully Poincaré covariance of the description, which allows to restore the expected symmetries, and the vanishing of dPDFs in the forbidden kinematical region, $x_{1}+x_{2}>1$, is achieved. In the analysis of dPDFs at the hadronic scale, the approximations of these quantities with a complete factorized ansatz, in the $x_{1}-x_{2}$ and $\left(x_{1}, x_{2}\right)-k_{\perp}$ dependences, are found to be violated, in agreement with previous results [13, 14]. Moreover, a pQCD analysis of the valence dPDFs, necessary to evaluate these quantities at higher energy scales with respect to the hadronic one where the CQM predictions are valid, has been performed. For the unpolarized quarks case dynamical correlations are suppressed in the small $x$ region, while double spin correlations are found to be still important. Further analysis, including into the scheme non perturbative sea quarks and gluons together with the evolution of dPDFs including the singlet sector contribution, fundamental in order to describe the dPDF at low $x$, are under way, as well as the study of the extraction of proton dPDFs from $p A$ collisions, along the line of Ref. [28].

\section{Acknowledgments}

This work was supported in part by the Research Infrastructure Integrating Activity Study of Strongly Interacting Matter (acronym HadronPhysic3, Grant Agreement n. 283286 and n. 283288) under the Seventh Framework Programme of the European Community, by the Mineco under contract FPA2010-21750-C02-01, by GVAPrometeo/2009/129, and by CPAN(CSD-00042). M.R. thanks the organizers of the conference for the invitation.

\section{References}

[1] T. Akesson, et al., Z. Phys. C 34, 163 (1987)

[2] J.R. Gaunt and W.J. Stirling, JHEP 1003, 005 (2010)

[3] M. Diehl, D. Ostermeier and A. Schafer, JHEP 1203, 089 (2012)

[4] A. V. Manohar and W. J. Waalewijn, Phys. Rev. D 85, 114009 (2012)

[5] P. Bartalini (ed.) and L. Fanò (ed.), arXiv:1003.4220 [hep-ex]

[6] S. Bansal, P. Bartalini, B. Blok, D. Ciangottini, M. Diehl, F. M. Fionda, J. R. Gaunt and P. Gunnellini et al., arXiv:1410.6664 [hep-ph].

[7] G. Aad, et al. [ATLAS Collaboration], New J. Phys. 15, 033038 (2013)

[8] N. Paver and D. Treleani, Nuovo Cim. A 70215 (1982)

[9] R. Kirschner, Phys. Lett. B 84, 266 (1979)

[10] V.P. Shelest, A.M. Snigirev and G.M. Zinovev, Phys. Lett. B 113, 325 (1982)

[11] M. Diehl, T. Kasemets and S. Keane, JHEP 1405, 118 (2014)

[12] T. Kasemets and P. J. Mulders, arXiv:1411.0726 [hep-ph].

[13] H.M. Chang, A.V. Manohar and W.J. Waalewijn, Phys. Rev. D 87, 034009 (2013)

[14] M. Rinaldi, S. Scopetta and V. Vento, Phys. Rev. D 87, 11, 114021 (2013)

[15] M. Traini, A. Mair, A. Zambarda and V. Vento, Nucl. Phys. A 614, 472 (1997)

[16] S. Scopetta and V. Vento, Eur. Phys. J. A 16, 527 (2003)

[17] A. Courtoy, F. Fratini, S. Scopetta and V. Vento, Phys. Rev. D 78, 034002 (2008)

[18] M. Rinaldi, S. Scopetta, M. Traini and V. Vento, arXiv:1409.1500 [hep-ph]. JHEP (2014) in press.

[19] B.D. Keister and W.N. Polyzou, Adv. Nucl. Phys. 20, 225 (1991)

[20] S.J. Brodsky, H.C. Pauli and S.S. Pinsky, Phys. Rept. 301, 299 (1998)

[21] F. Cardarelli, E. Pace, G. Salmè and S. Simula, Phys. Lett. B 357, 267 (1995) 
[22] P. Faccioli, M. Traini and V. Vento, Nucl. Phys. A 656, 400 (1999)

[23] S. Boffi, B. Pasquini and M. Traini, Nucl. Phys. B 649, 243 (2003)

[24] S. Boffi, B. Pasquini and M. Traini, Nucl. Phys. B 680, 147 (2004)
[25] M. Traini, Phys. Lett. B 707, 523 (2012)

[26] M. Traini, Phys. Rev. D 89, 034021 (2014)

[27] W. Broniowski and E.R. Arriola, Few Body Syst. 55, 381 (2014)

[28] S. Salvini, D. Treleani and G. Calucci, Phys. Rev. D 89, 016020 (2014) 